\title{
MODELO PROPUESTO PARA LA IMPLEMENTACIÓN DE LA METODOLOGÍA SMED EN UNA EMPRESA DE ALIMENTOS DE SANTIAGO DE CALI
}

\author{
Proposed MOdEL FOR THE IMPLEMENTATION OF THE SMED METHODOLOGY \\ IN A FOOD COMPANY IN SANTIAGO DE CALI
}

Jairo Arboleda Zúñiga*

Fabián M. Rubiano del Chiaro**

Recibido: 14 de agosto de 2017

Aceptado: 9 de octubre de 2017

\section{Resumen}

En este artículo se propone aplicar el método SMED en el proceso de cambio de moldes en una máquina de conos de helados, propiedad de una empresa de alimentos ubicada en Santiago de Cali. Actualmente, la flexibilidad de los procesos es un elemento fundamental para mejorar la productivi$\mathrm{dad}$, factor que se puede constituir en fuente generadora de ventajas competitivas en el mercado. La metodología de estudio consiste en la identificación de una de las máquinas más usada en la fabricación de conos, a través de análisis de tiempos perdidos y eficiencia; una vez identificada la máquina, se define cuál es el cambio más significativo y de esta manera se analizan los hallazgos obtenidos por medio de la técnica y se presenta la propuesta a ser considerar en su implementación, que sirve de base para mejorar las otras máquinas de la línea de producción. Existen aplicaciones del SMED en procesos de cambio de moldes en máquinas inyectoras, pero se desconoce si este método se ha enfocado especificamente en la elaboración de conos; por consiguiente, este artículo puede servir de referencia a otras empresas en las que la aplicación del SMED se podría hacer de forma idéntica o similar.

Palabras clave: metodología SMED, proceso de cambio de moldes, lean manufacturing en empresa de helados.

\section{Abstract}

This article proposes to apply the SMED method in the process of changing molds in an ice cream cones machine, owned by a food company located in Santiago de Cali. Currently, the flexibility of the processes is a fundamental element to improve productivity, a factor that could become a source for generating competitive advantages in the market. The study methodology consists in the identification of one of the most used machines in the manufacture of cones, through analysis of lost times and efficiency; once the device has been identified, the most significant change is defined and in this way the findings obtained by means of the technique are analyzed, and the proposal to be considered in its implementation is presented, which serves as the basis for improving the other machines the production line. There are applications of the SMED in processes of

\footnotetext{
* Ingeniero industrial, magíster en Ingeniería Industrial. Investigador del Grupo-IDEAS, docente de la Facultad de Ingeniería, Universidad Santiago de Cali, Cali, Colombia. ORCID:http://orcid.org/0000-0003-4045-9396. jarboledaz@usc.edu.co

${ }^{* *}$ Ingeniero Industrial, Universidad Santiago de Cali, Cali, Colombia. ORCID:http://orcid.org/0000-0001-9484-7891. fabian.rubiano00@usc.edu.co
} 
change of molds in injection molding machines, but it is unknown if this method has explicitly focused on the elaboration of cones; therefore, this article might work as a reference for other companies in which the application of the SMED may be done in an identical or similar way.

Keywords: SMED methodology; the process of change of molds; lean manufacturing in an ice cream company.

\section{INTRODUCCIÓN}

La organización en estudio es una pequeña empresa industrial (fundada en Cali en 1987) que ha creado cuarenta empleos directos. Su visión de crecimiento y expansión está enfocada a la producción y comercialización de conos de galleta para helados. La empresa cuenta con equipos de alta tecnología y personal calificado. La demanda que presenta la compañía y sus futuros proyectos de inversión para nuevos productos es lo que ha llevado a la gerencia a pensar en nuevas alternativas que generen una alta y oportuna capacidad de respuesta frente al mercado, para así consolidarse como una empresa productiva y competente.

En el año 2016, la empresa tuvo altos índices de desperdicio de tiempo en el área de producción, lo que ocasionó una reducción considerable en las ventas, puesto que las referencias producidas estaban por debajo de lo demandado (e, incluso, se presentó la ausencia de algunas referencias), tanto así que al finalizar el año no generó las utilidades presupuestadas.

Luego de analizar los resultados obtenidos en el año, el equipo directivo de la empresa consideró que una de las principales causas que afectaban el proceso fue el desperdicio de tiempo en la preparación y mantenimiento de las máquinas, en este caso, el tiempo empleado en el montaje y desmontaje de moldes.

En la tabla 1 se muestra el porcentaje de tiempo perdido en cada una de las máquinas de producción el año 2016, y en la tabla 2, el porcentaje de eficiencia para el mismo año:

Tabla 1. Porcentajes de tiempo perdido en las máquinas de producción (2016)

\begin{tabular}{llllll}
\hline \multicolumn{7}{c}{ Máquinas de producción de productos } \\
\hline & MQ1 & MQ2 & MQ3 & \multicolumn{1}{c}{ MQ4 } & \multicolumn{1}{c}{ MQ5 } \\
\hline Horas perdidas & 216 & 348 & 518 & 221 & 273 \\
Horas programadas & 2844 & 2376 & 6777 & 2932 & 3440 \\
\% de tiempo perdido & 7.59 & 14.65 & 7.64 & 7.54 & 7.93 \\
\hline
\end{tabular}

Fuente: área de producción, empresa en estudio.

Tabla 2. Porcentajes de eficiencia de las máquinas de producción (2016)

\begin{tabular}{lccccc}
\hline \multicolumn{5}{c}{ Máquinas de producción de productos (miles) } \\
\hline & MQ1 & MQ2 & MQ3 & MQ4 & MQ5 \\
\hline Producción real & 7955 & 4683 & 25716 & 8797 & 10521 \\
& und & und & und & und & und \\
Producción esperada & 10.15 & 7.460 & 28.800 & 10.735 & 13.219 \\
& und & und & und & und & und \\
\% de eficiencia & 78.3 & 62.8 & 89.3 & 81.9 & 79.6 \\
\% de und. rechazadas & 16.3 & 56.4 & 6.0 & 13.2 & 14.3 \\
\hline
\end{tabular}

Fuente: área de producción, empresa en estudio. 
Por lo tanto, en este artículo se elabora una propuesta de aplicación de la metodología SMED en nuestro caso de estudio, la cual se realiza en el proceso de cambio de moldes en la maquina 2, puesto que esta máquina produce lotes pequeños varias referencias (por lo que se requiere reducir al máximo los tiempos de cambio) y además tiene un mayor porcentaje de desperdicio de tiempo, por lo que es menos eficiente y tiene más porcentaje de rechazo que las otras máquinas de producción de la empresa.

$\mathrm{Al}$ respecto de la producción de lotes pequeños, Mcintosh, Culley, Mileham y Owen (2000) aseguran que:

[...] un alistamiento rápido es un componente fundamental de la filosofía de manufactura moderna, por lo que mejorar su desempeño es clave para permitir la fabricación de lotes pequeños; ya que las intervenciones se vuelven tan cortas que deben hacerse económicamente viable; es decir realizar múltiples paradas para producir una variedad de productos, debe ser rentable. (p.37)

La minimización del costo del cambio de útiles y, en consecuencia, la maximización del aprovechamiento de los medios de producción dependen en gran medida de que el diseño de las máquinas se haga no solo considerando la velocidad de producción, sino también la necesidad de realizar un cambio rápido de útiles (Rodríguez y Cárcel,2014, p. 69).

Según Rodríguez (2003), las técnicas destinadas a mejorar el proceso de cambio de útiles son escasas, a pesar de la relevancia que ha adquirido este proceso, sobre todo en entornos de fabricación flexible.

\section{FundaMENTOS TEÓRICOS}

\section{Proceso de moldeo de conos de helado}

El proceso de moldeo de conos en máquinas consiste en moldear una mezcla de masa puesta por una unidad vertedora en las mitades inferiores de los moldes de cocción para hornear. Los moldes deben estar calientes y cerrados para circular en el quemador de gas; tras un ciclo, las partes superiores de los moldes se abren y enseguida se obtiene una pieza moldeada de un cono de helado (I. M. A. R., 2013).

La producción de conos de helado por medio de máquinas de moldeo representa ventajas con respeto a otras maneras de producirlo, entre ellas: rapidez de fabricación, alto nivel de producción, gran diversidad de productos en sus moldes y bajos costos de producción. La calidad del producto y la estabilidad del proceso de moldeo están directamente relacionadas con la mezcla de masa, la capacidad y limitaciones de la maquinaria, el diseño de moldes, las características de la materia prima y, por supuesto, la experiencia de los operadores.

En el pasado, los cambios de moldes:

[...] estaban basados en diferentes estrategias como usar personas con habilidades especiales para realizar cambios de moldes, producción de grandes lotes o fabricar de acuerdo al tamaño económico de lote; en las que implicaban destreza, los operadores tenían que tener habilidades necesarias para montar y desmontar los moldes a cambiar. Este tipo de estrategia tenía el inconveniente de necesitar trabajadores especializados en los cambios y no siempre se era eficiente. (Lerma, 2014, p.14)

\section{Lean manufacturing}

Socconini (2008) define lean manufacturing como: 
Un proceso continuo y sistemático de identificación y eliminación del desperdicio o excesos, entendiendo como exceso toda aquella actividad que no agrega valoren un proceso, pero sí costo y trabajo, está eliminación sistemática se lleva a cabo mediante trabajo con equipos de personas bien organizados y capacitados. (p.75)

Desde el enfoque sistémico, Shah y Ward (2007) definen el lean manufacturing como: "un sistema integrado sociotecnológico cuyo objetivo principal es eliminar desperdicios, minimizando o reduciendo suplidores y variabilidad interna" (p.792).

Gisbert (2015) propone una de las definiciones más actuales de lean manufacturing:

[...] una filosofía que se apoya en una serie de técnicas cuya finalidad es la mejora de la productividad de la empresa, soportada por un conjunto de herramientas que: ayudarán a eliminar todas las operaciones que no agreguen valor al producto, servicio y a procesos; aumentarán el valor de cada actividad realizada, eliminando lo que no se requiere; reducirán desperdicios y mejorarán las operaciones, basándose siempre en el respeto al trabajador y obtendrán así mejoras tangibles, medibles y significativas de la competitividad. (p. 42)

El lean manufacturing es una teoría fundamental para el mejoramiento de la productividad y la competitividad de las empresas de manufactura y servicios, que tiene el objetivo de "generar una nueva cultura de la mejora basada en la comunicación y en el trabajo en equipo; para ello es indispensable adaptar el método a cada caso concreto" (Varela, Ramírez y Gómez, 2015, p.190).

Para Melton (2014), el lean manufacturing puede ser aplicado a todos los aspectos de la cadena de suministro y debe ser visto dentro de la organización como una de las herramientas que genera un gran número de beneficios de manera sostenible.

Hernández y Vizán(2013) desarrollaron una gráfica para visualizar rápidamente la filosofía que encierra esta teoría y las técnicas disponibles para su aplicación, que se explica "utilizando una casa porque esta constituye un sistema estructural que es fuerte siempre que los cimientos y las columnas lo sean; una parte en mal estado debilitaría todo el sistema" (p.46). La figura 1 representa una adaptación actualizada de esta "casa".

Todos los elementos de esta casa se construyen a través de la aplicación de múltiples técnicas que han sido divididas según se utilicen para el diagnóstico del sistema a nivel operativo, o como técnicas de seguimiento. Estas técnicas pueden implantarse de forma independiente o conjunta, atendiendo a las características específicas de cada caso (Hernández y Vizán, 2013, p. 48).

Vilana (2010) sostiene que en lean manufacturing:

[...] lo único que importa producir es lo que el cliente realmente percibe como valor, por lo que un aspecto esencial es entender quién es el cliente (interno o externo) y qué quiere, es decir comprender sus necesidades, expectativas y requerimientos e incorporarlos a los procesos de trabajo. Cada tarea, función o actividad debe añadir valor, hay que identificar el camino de valor con el fin de eliminar el desperdicio [muda], desde que se introduce la materia prima y se transforma, hasta que se entrega el producto terminado al cliente. (pag. 11) 


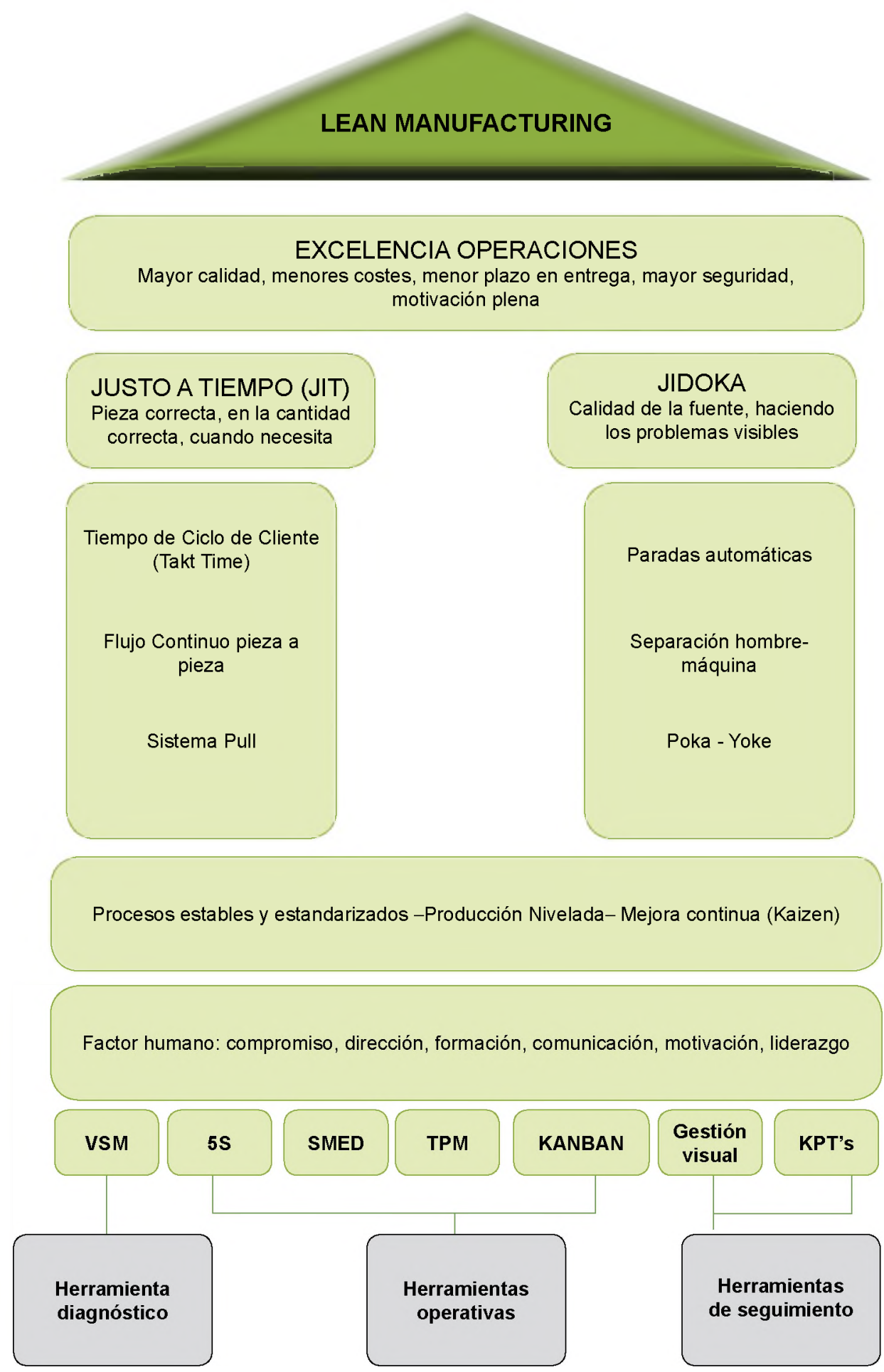

Figura1. Representación de las técnicas lean manufacturing.

Fuente: Hernández y Vizán (2013). 


\section{Metodología SMED}

En 1950 el japonés Shigeo Shingo (1909-1990) (citado en Madariaga, 2013) comenzó a trabajar en la reducción de los tiempos de cambio de las prensas. A lo largo de treinta años, desarrolló una metodología a la que denominó SMED (single minute exchange of die), cuya traducción es "cambio de útiles en pocos minutos". Shingo colaboró con Toyota durante más de 25 años como consultor $\mathrm{y}$ formador.

Shingo (1985) introduce la idea de que en general cualquier cambio de máquina o alistamiento del proceso:

[...] debería durar no más de 10 minutos, de ahí la frase single minute (minutos en un solo dígito), haciendo que las operaciones de preparación sean más rápidas y simples, el SMED ayuda a la empresa a producir en pequeños lotes. (p. 85)

\section{Para Shingo (1989), la necesidad del SMED surge:}

[...] cuando el mercado demanda una mayor variedad de producto y los lotes de fabricación deben ser menores; en este caso para mantener un nivel adecuado de competitividad, o se disminuye el tiempo de cambio o se siguen haciendo lotes grandes y se aumenta el tamaño de los almacenes de producto terminado, con el consiguiente incremento de los costos; esta técnica está ampliamente validada y su implantación es rápida y altamente efectiva en la mayor parte de las máquinas e instalaciones industriales. (p.89)

Una ventaja muy importante al reducir los tiempos de preparación a un solo dígito es que la empresa:

Puede pasar de trabajar contra almacén a fabricar bajo pedido, dado que para algunas fábricas la inversión en el inventario de producto acabado es el mayor activo, su conversión en efectivo puede servir para financiar otras inversiones o reducir deudas. (Velasco, 2013, p. 320).

Villaseñor y Galindo(2007) describen las siguientes etapas para la aplicación del SMED:

1. Etapa preliminar: No se distinguen las preparaciones internas y externas. En las operaciones de preparación tradicionales, se confunde la preparación interna con la externa y lo que puede realizarse externamente se hace internamente, lo que trae como consecuencia que las máquinas estén paradas durante grandes periodos de tiempo.

2. Primera etapa: Separación de la preparación interna y externa. El paso más importante en la realización del sistema SMED es la diferenciación entre la preparación interna y la externa. Todo el mundo está de acuerdo en que la preparación de piezas, el mantenimiento de los dados, herramientas y ciertas operaciones, no se deben hacer mientras la máquina esta parada. Sin embrago, esto ocurre con frecuencia.

3. Segunda etapa: Conversión de la preparación interna en externa. La segunda etapa comprende dos conceptos importantes: reevaluar las operaciones, para ver si algunos pasos están erróneamente considerados como internos y buscar formas para convertir esos pasos internos en externos.

4. Tercera etapa: Perfeccionamiento de todos los aspectos de la operación de preparación. Aunque el nivel de los diez minutos se puede alcanzar algunas veces, simplemente convirtiendo la preparación interna en externa, no es así en la mayoría de los casos. Esta es la razón por la cual se debe concentrar esfuerzos para perfeccionar todas y cada una de las operaciones elementales que constituyen las preparaciones interna y externa. Consecuentemente, la tercera etapa necesitara un análisis detallado de cada operación. (Villaseñor y Galindo, 2007, p. 91) 
Algunos investigadores, como Goubergen y Landeghem (2002), sugieren que con SMED es fácil lograr reducciones de tiempo hasta del $90 \%$; sin embargo, la revisión de la literatura no evidencia ejemplos descriptivos que demuestren estos resultados en casos reales de implementación.

La aplicación de la metodología de Shingo generalmente se traduce en dos beneficios principales: aumentar la capacidad de fabricación y mejorar la flexibilidad del equipo. Eso permite trabajar con tamaños de lote más pequeños, lo que crea un flujo de materiales eliminando la espera (Coimbra, 2009).

Para Singh y Khanduja (2009), la metodología SMED podría ser ineficaz:

[...] si no se tienen en cuenta algunos requisitos previos a su ejecución; al ser una herramienta de cambio cultural es importante tomar conciencia de la importancia que tiene para la empresa y sus actividades la disminución de los tiempos de preparación, preparar a los empleados mediante capacitación y entrenamiento a los efectos de incrementar la productividad y reducir los costos mediante la reducción en los tiempos de preparación, hacer un cambio de paradigmas, terminando con las creencias acerca de la imposibilidad de disminuir radicalmente los tiempos de preparación. (p. 105)

\section{MATERIALES Y MÉTODOS}

Actualmente la empresa cuenta con cinco máquinas de producción marca I.M.A.R., máquinas automáticas diseñadas para la cocción y la producción de productos alimenticios a base de harina (conos de helado).

\section{Descripción de máquina}

Cada máquina está compuesta por tres módulos:

- Cuerpo de la máquina con calefacción de gas y tracción eléctrica y 21 moldes (hembra fundido, latón cromado macho, peso aproximado $37 \mathrm{~kg} \mathrm{c} / \mathrm{u}$ ) para la cocción del producto.

- Bomba de bombeo/bomba de inyección.

- Apilamiento de canaleta (tobogán) y se deslizan los conos. En este módulo se cuenta, apila y queda listo el embalaje del producto.

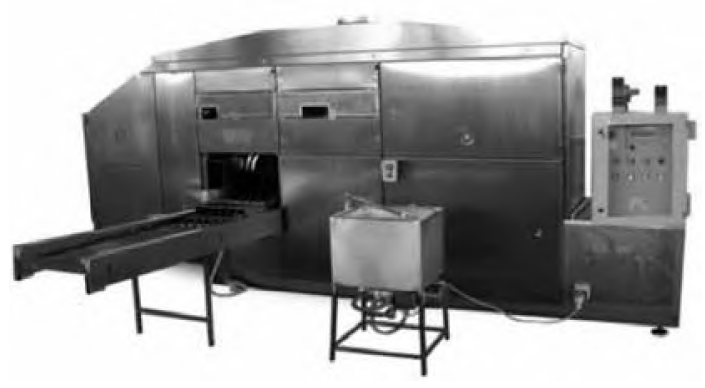

Figura 2. Representación de máquina de conos

Fuente: I. M. A. R. (2013).

\section{Funcionamiento de máquina}

Su funcionamiento se basa en que su bomba de inyección automática que está conectada a un surtidor (flauta) equipado con boquillas. Este sistema inyecta la cantidad fija de masa directamente en el molde para hornear. Los moldes, que se calientan mediante un quemador de gas colocado en la parte inferior de la máquina, están posicionados en cadena y su movimiento es continuo. Una 
vuelta completa del molde de la cadena corresponde a un ciclo de cocción completa; al final del ciclo de cocción, se abre el molde y el producto cae naturalmente en el tobogán.

\section{Cambio de moldes y mantenimiento}

El proceso de cambio de moldes es realizado por el único operario de mantenimiento a la fecha (trabaja en un turno de 8 horas diarias); este cambio empieza cuando el jefe de producción da la orden de desmontar el molde, después de haber terminado el último lote producido de unas de las referencias que se fabrican en esa máquina, para montar el molde de la nueva referencia a fabricar.

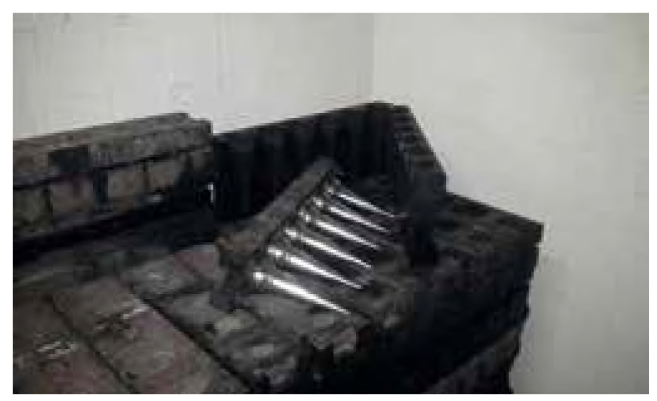

Figura 3. Representación de moldes de conos Fuente: I. M. A. R. (2013).

\section{Estudio de métodos y tiempos}

A finales del cuarto trimestre del año 2016, se observó que en la maquina 2 es la que se realizaban más cambios de moldes para producir cuatro diferentes referencias en sus periodos de producción. Esta máquina produce alrededor de 16261 unidades diarias. De cada referencia se elaboran entre tres y cuatro lotes, lo que conlleva realizar aproximadamente cinco cambios bimestrales, debido a que las otras máquinas están produciendo las demás referencias del portafolio de venta o se encuentran en estado de mantenimiento preventivo.

Los tiempos en el proceso de cambio de moldes fueron tomados con cronómetro y consignados en un formato establecido por la empresa, en el que también se consignó toda la información pertinente sobre el método de estudio, las herramientas utilizadas, la descripción de las operaciones, el departamento donde se realiza la operación y las condiciones de trabajo que prevalecen. Este análisis fue realizado bajo los conceptos planteados por Niebel y Freivalds (2004) para el estudio de métodos y tiempos.

Además, se pidió ayuda a dos analistas que tienen experiencia y conocimiento necesario del proceso de fabricación de conos, quienes fueron repartidos en cada uno de los turnos y estuvieron presentes al momento de hacer el cambio de referencia en la maquina 2 durante el periodo de estudio, con el fin de recolectar la mayor información posible en las tres tomas de tiempo.

Por lo tanto, se aplicó a la maquina 2 la metodología SMED para la reducción de pérdida de tiempo a la preparación y mantenimiento de la máquina.

\section{Resultados Y discusión}

\section{Etapa preliminar}

Este estudio de métodos y tiempos permitió hacer un análisis de las operaciones que se realizan en el cambio de los 21 moldes realizado por un operario, desglosándolas actividades lo mejor posible 
y determinando el tiempo que requiere cada una de ellas, además de los utillajes y herramientas que se precisan.

En la tabla 3 se presentan de manera resumida las actividades involucradas en el proceso:

Tabla 3. Resumen de actividades en el proceso de cambio de moldes

\begin{tabular}{llll}
\hline Figura & Descripción & \multicolumn{1}{c}{ Cant. } & \multicolumn{1}{c}{ T. OP. (min) } \\
\hline & Operaciones & 366 & $518: 05$ \\
& Inspecciones & 45 & $77: 10$ \\
& Transportes & 21 & $28: 21$ \\
& Almacenajes & 21 & $24: 30$ \\
& Demoras & 4 & $1295: 15$ \\
\hline & TOTAL & 457 & $1943: 21$ \\
\hline
\end{tabular}

El tiempo total de la operación es de 1943 min $21 \mathrm{~s}$.

Podemos identificar que al operario realiza todas las actividades en 4 turnos de 8 horas, es decir, 4 días para terminar el proceso de cambio de moldes e iniciar la producción de alguno de los ítems programado.

\section{Primera etapa: separación de operaciones internas y externas}

Una vez hecho el desglose de las operaciones en el proceso de cambio de molde, el siguiente paso es identificar qué operaciones se pueden realizar mientras la máquina está en funcionamiento y cuáles se pueden eliminar, en la medida en que no aportan valor a la operación (Villaseñor y Galindo, 2007).

Para este análisis también utilizamos el diagrama de operaciones conjuntas, en el que identificamos en cuánto tiempo el operario realiza actividades internas que están en el proceso de cambio de moldes con la máquina parada, cuando estas podrían realizarse con la máquina en operación antes o después del cambio de moldes.

En la tabla 4 se muestran las operaciones de preparación interna que se pueden cambiar a externas:

Tabla 4. Operaciones de preparación interna

\begin{tabular}{|c|c|c|}
\hline Figura & Descripción & T. OP (min) \\
\hline & Trasladar el primer molde hasta la máquina & $1: 21$ \\
\hline & Verificar el estado operativo de la máquina & $1: 45$ \\
\hline & Reparar eslabón en taller & $345: 28$ \\
\hline & Cambiar bujes, carcasas en taller & $34: 36$ \\
\hline & Abrir y quitar tapas de la maquina & $2: 55$ \\
\hline & Alistamiento de herramientas & 3:00 \\
\hline & $\begin{array}{l}\text { Buscar y ubicar estiba en máquina para inicio } \\
\text { de cambio }\end{array}$ & $0: 45$ \\
\hline
\end{tabular}




\begin{tabular}{llc}
\multicolumn{1}{c}{ Descripción } & T. OP (min) \\
\hline & $\begin{array}{l}\text { Colocar y cerrar tapas de la maquina } \\
\text { mientras arranca a operar }\end{array}$ & $2: 55$ \\
& $\begin{array}{l}\text { Retirar estiba } \\
\text { Trasladarse a buscar piezas pequeñas de } \\
\text { repuesto al taller }\end{array}$ & $1: 51$ \\
& $\begin{array}{l}\text { Trasladar el ultimo molde al taller } \\
\text { Almacenar el ultimo molde en el taller }\end{array}$ & $1: 21$ \\
& TOTAL & $397: 52$ \\
\hline
\end{tabular}

\section{Segunda etapa: conversión de operaciones internas en externas}

Las operaciones de preparación interna descritas anteriormente se convierten en operaciones de preparación externa, que pasan a realizarse mientras la máquina está en marcha (Villaseñor y Galindo, 2007).

Para realizar esta conversión se realizó un análisis individual de las operaciones en conjunto con el jefe de producción y mantenimiento, en la que se pudo balancear la carga del operario sin requerir inversión alguna, solo un reordenamiento de las operaciones realizadas. Esto implica un gran desafío, pues para que el operario realice esta nueva secuencia de actividades se requiere una capacitación frecuente.

Tras la conversión de operaciones internas en externas, el proceso de cambio de moldes queda de la siguiente manera:

Tabla 5. Resumen de actividades en el proceso de cambio de moldes (primera mejora)

\begin{tabular}{rlcc}
\hline Figura & Descripción & Cant. & T. OP. (min) \\
\hline & Operaciones & 361 & $507: 45$ \\
& Inspecciones & 44 & $75: 15$ \\
& Transportes & 18 & $24: 08$ \\
& Almacenajes & 20 & $23: 20$ \\
\hline & Demoras & 2 & $915: 11$ \\
\hline & TOTAL & 445 & $1545: 39$ \\
\hline
\end{tabular}

Tras la mejora implantada, se redujo el tiempo de preparación en un $20.47 \%$.

Tiempo total del proceso de cambio de moldes antes de la aplicación del SMED: 1943 min $21 \mathrm{~s}$. Tiempo total del proceso de cambio de moldes tras la segunda etapa del SMED: 1545 min $39 \mathrm{~s}$.

$$
\% \text { de tiempo reducido }=\frac{(1943 \min 21 s-1545 \min 39 s)}{1943 \min 21 s} * 100
$$

$\%$ de tiempo reducido $=20.47 \%$ 


\section{Tercera etapa: perfeccionar las operaciones internas y externas}

El siguiente paso para la reducción del tiempo de cambio de referencia es mejorar y reducir el tiempo de las tareas externas (Villaseñor y Galindo, 2007).

Si en esta etapa se dispusiera de un equipo de mantenimiento consolidado o, por lo menos, de un operario de planta capacitado que apoyara las demás actividades externas que demoran la operación de cambio de moldes, se reduciría el tiempo que se pierde cuando se espera para continuar la operación de un día a otro, pues sería una operación continua sin tiempo de espera para seguir el proceso de montaje del siguiente molde. Las actividades que se pueden suprimir al disponer de dos operarios mecánicos, uno para cada turno, se muestran en la tabla 6:

Tabla 6. Operaciones de preparación externa que se pueden suprimir

\begin{tabular}{|c|c|c|}
\hline Figura & Descripción & T. OP.(min) \\
\hline & $\begin{array}{l}\text { Suspensión de operación hasta el } \\
\text { día siguiente }\end{array}$ & 900 \\
\hline & TOTAL & 900 \\
\hline
\end{tabular}

Con esta mejora aplicada, el estudio de la operación generaría los siguientes resultados:

Tabla 7. Resumen de actividades en el proceso de cambio de moldes (segunda mejora)

\begin{tabular}{llcc}
\hline & Descripcion & Cant. & T. OP. (min) \\
\hline & Operaciones & 361 & $507: 45$ \\
& Inspecciones & 44 & $75: 15$ \\
& Transportes & 18 & $24: 08$ \\
& Almacenajes & 20 & $23: 20$ \\
\hline & Demoras & 1 & $15: 11$ \\
\hline & TOTAL & 444 & $645: 39$ \\
\hline
\end{tabular}

Con la aplicación de la tercera etapa del SMED se redujo el tiempo del proceso en $58.23 \%$.

Tiempo total del proceso de cambio de moldes tras la segunda etapa del SMED: 1545 min $39 \mathrm{~s}$.

Tiempo total del proceso de cambio de moldes tras la tercera etapa del SMED: $645 \mathrm{~min} 39 \mathrm{~s}$.

$$
\% \text { de tiempo reducido }=\frac{(1545 \min 39 s-645 \min 39 s)}{1545 \min 31 s} * 100
$$

$\%$ de tiempo reducido $=58.23 \%$

El tiempo de preparación en el proceso de cambio de moldes se redujo un $66.8 \%$, que equivale a $1297 \mathrm{~min} 42 \mathrm{~s}$.

Tiempo total del proceso de cambio de moldes antes de la aplicación del SMED: 1943 min $21 \mathrm{~s}$.

Tiempo total del proceso de cambio de moldes después de la aplicación del SMED: 645 min $39 \mathrm{~s}$. 


$$
\% \text { de tiempo reducido }=\frac{(1943 \min 21 s-645 \min 39 s)}{1943 \min 21 s} * 100
$$

$\%$ de tiempo reducido $=66.78 \%$

Con la aplicación del SMED se puede lograr una reducción de $1297 \mathrm{~min} 42 \mathrm{~s}$ en el proceso de cambio de moldes, que en un año se ahorrarían un total de 38931 minutos.

\section{$1297 \mathrm{~min} 42 \mathrm{sx} 5 \mathrm{cambios} /$ bimestrales $\mathrm{x}$ 6bimestres $=38931 \mathrm{~min}$}

Que equivalen a la producción de aproximadamente 439623 unidades/año. Y además un ahorro de $\$ 3041000$ al año

Teniendo en cuenta todos estos resultados, la implementación del SMED en la maquina 2 implicaría que el tiempo de preparación sea menor; por lo tanto, aumenta la capacidad de la máquina y su eficiencia (ver figura 4).

\section{Eficiencia de máquina 2}

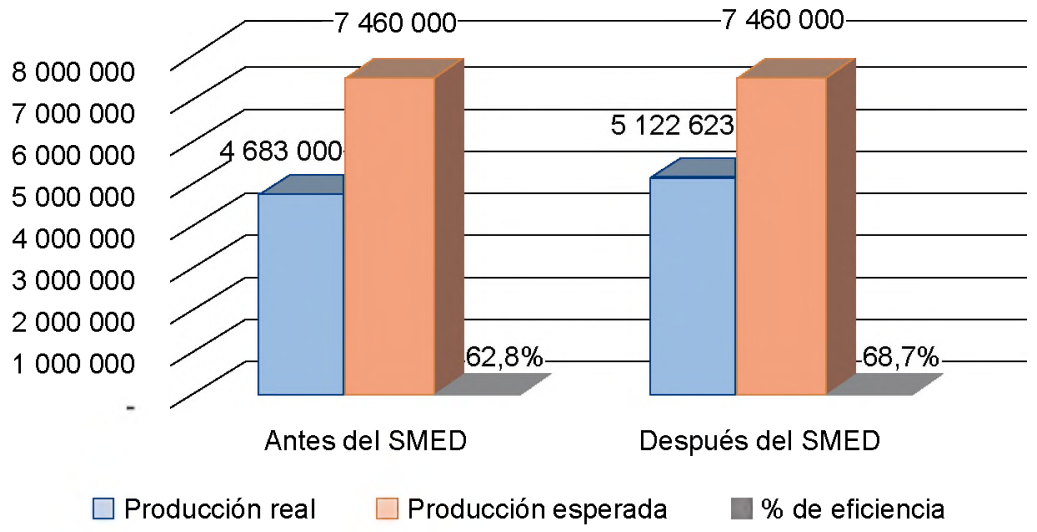

Figura 4. Representación de eficiencia.

El porcentaje de eficiencia de la línea se incrementaría en un $5.94 \%$. Con la técnica SMED se pueden identificar muchas actividades ineficientes que no aportan valor al proceso.

La empresa en estudio puede aplicar la metodología SMED a todas sus demás máquinas de producción -así como lo demuestran los resultados- y conseguir mejores beneficios, ya que una mayor reducción en los tiempos de preparación incrementaría la productividad y la eficiencia de la empresa.

Según Mcintosh et ál. (2000), la disminución de los tiempos de cambio de moldes permite la disminución en el tamaño de los lotes, lo que hace posible la reducción de los inventarios en proceso y, por consiguiente, es factible reducir los tiempos de ciclo; esto le permite dar a la empresa una respuesta más rápida a los clientes, lo que elimina la necesidad de mantener altos inventarios de productos terminados.

En la actualidad hay muchas empresas que han implementado la metodología SMED y han logrado posicionarse en el mercado en cuanto a productividad y competitividad, gracias a que apuntaron a la reducción en los tiempos de preparación, que consideraban era la principal causa de desperdicio de tiempo y pérdidas financieras para la organización. 
A continuación se mencionan algunos casos de éxito en empresas colombianas:

La empresa Familia Sancela de Cajicá aplicó la metodología SMED en una máquina empacadora de papel higiénico; el proyecto generó un incremento de $\$ 1037760000$ al año en producto terminado (Cárdenas y Hernández, 2008).

Papeles Nacionales S. A. aplicó la metodología SMED para el cambio de bobina en una máquina rebobinadora de papel higiénico; con este cambio la compañía logró reducir el tiempo de la operación en un 32 \% (183 segundos con SMED versus 270 segundos sin SMED) (Rojas y Cortéz, 2014)

Sobre empresas internacionales, se conocen algunos casos destacables:

Amcore Pet Packaging (Michigan, EE. UU.) es líder en el negocio de envases plásticos; su planta elabora nueve millones de preformas al día por medio de máquinas de soplado y moldeo por inyección. Esta empresa obtuvo resultados espectaculares con el SMED: redujo los tiempos de cambio en un $54 \%$, lo que generó ahorros de $£ 60000$ para la empresa (Rowlands, 2006).

La empresa Alfa (Portugal), dedicada a la producción de espuma de poliuretano y poliéster para varios mercados, empleó la metodología SMED en las máquinas de corte de los rollos de espuma y logró reducir el tiempo de preparación de 74 a 40 min, lo que representó una mejora del $65 \%$; igualmente, el coste unitario del producto total se redujo en un $13 \%$ (Carrizo y Torres, 2013).

\section{Conclusiones}

Con la implementación de la metodología SMED la empresa podrá reducir los tiempos de preparación en el proceso de cambio de moldes de la maquina 2 en un $66.77 \%$ (439 623 unidades/año, aproximadamente), lo que generaría mayor capacidad de respuesta y un aumento de sus utilidades. Igualmente, la empresa obtendría un ahorro de por lo menos $\$ 3041000$ al año (teniendo en cuenta el salario del presente año), sin olvidar que su costo de producción bajaría por lo menos $\$ 1$ por unidad, solo considerando la máquina en la que se aplicaría la mejora del proceso.

La empresa en estudio cuenta con cinco máquinas de producción y maneja más de diez referencias, por lo que es necesario realizar muchos cambios en las máquinas; de tal manera que la implementación de la metodología SMED en todas sus máquinas podría generar importantes aportes a la empresa, como:

- Mayor flexibilidad sin necesidad de mantener grandes inventarios.

- Mayor capacidad de respuesta en el servicio al cliente, en la medida en que puede alcanzar ciclos más cortos en fabricación por la introducción de pequeños lotes de producción.

- Disminución en los tiempos de preparación o alistamiento y tiempos ociosos en las máquinas y los equipos.

- Mejoramiento en el desempeño del indicador de productividad, al incrementarse el output y disminuirse la utilización de los recursos productivos.

La técnica SMED es una metodología de fácil aplicación que se adapta a cualquier empresa, y las empresas del sector alimenticio no son la excepción para su aplicación. Lo importante es combinar SMED con las demás técnicas de mejora continua de lean manufacturing, para obtener mejores resultados y poder lograr que la empresa sea más competitiva en el mercado global.

Disponer de una ventaja a nivel de mejora de procesos y crecer competitivamente son factores fundamentales para el crecimiento de una empresa, ya que bajar los costos operativos es una de las metas de cualquier organización, más en el caso de mercados emergentes como el colombiano, donde cualquier peso puede proyectarse para futuros proyectos de inversión. 


\section{REFERENCIAS}

Cakmakci, M., Karasu, M., y Cakiroglu, M. (2014). Improvement of changeover times via Taguchi empowered SMED/case study on injection molding production. Measurement, 47, 741-748.

Cárdenas, I., y Hernández, J. (2008). Aplicación del SMED en una máquina empacadora de papel bigiénico en la planta de conversión de papel higiénico de Familia Sancela en Cajicá (tesis). Recuperado de http://intellectum.unisabana.edu.co/bitstream/handle/10818/7002/125072. pdf?sequence $=1$

Carrizo, A., y Torres, P. (2013). Implementation of the Single Minute Exchange of Die (SMED) Methodology in Small to Medium-sized Enterprises: A Portuguese Case Study. International journal of management, 30(1), 66-87.

Coimbra A., E. (2009). Total flow management:achieving excellence with kaizen and lean supply chains. [s. d.]: Kaizen Institute.

Gisbert, V. (2015). Lean manufacturing. Qué es y qué no es, errores en su aplicación e interpretación más usuales. $3 C$ Tecnología, 13(4), 42-52.

Goubergen, D., y Landeghem, H. (2002). Rules for integrating fast changeover capabilities into new equipment design. Robotics and Computer Integrated Manufacturing, 18(3), 205-214.

Hernández, J., y Vizán, A. (2013). Lean manufacturing: conceptos, técnicas e implantación. Madrid, España: Fundación EOI. Recuperado de http://www.eoi.es/savia/documento/eoi80094/lean-manufacturing-conceptos-tecnicas-e-implantacion

I. M. A. R. (2013). Ice Cream Cones Baking Machines. Roma, Italia: I. M. A. R. Recuperado de http://www.imaritaly.com/

Lerma, J. (2014). Manual advanced thermoplastic processing by injection: scientific injection molding. Barcelona: 9Disseny.

Madariaga, F. (2013). Lean manufacturing: Exposición adaptada a la fabricación repetitiva de familias de productos mediante procesos discretos. [s. d.]: Bubok Publishing.

Mcintosh, R., Culley, S., Mileham, A., y Owen, G. (2000). A critical evaluation of Shingo`s SMED (Single Minute Exhange of Die) methodology. International Journal Of Production Research, 38(11), 2377-2395.

Melton, T. (2004). To lean or not to lean? (that is the question). The chemical Engineer, 759, 34-37.

Niebel, B., y Freivalds, A. (2004). Ingeniería industrial: métodos, estándares y diseño del trabajo (11 ed.). México: Alfaomega.

Rodríguez, M., y Cárcel, F. (2014). Methodology and considerations for setup operations in production. Tecnica Industrial, 68-71.

Rodríguez, M. (2003). El proceso de cambio de útiles. La flexibilidad de una fábrica. Madrid: Fundacion Confemetal.

Rojas, L., y Cortéz, C. (2014). Aplicación de la metodología SMED para el cambio de bobina de semielaborado en una máquina rebobinadora de papel bigiénico en la empresa Papeles $N a-$ cionales S. A. (tesis). Recuperado de http://repositorio.utp.edu.co/dspace/bitstream/ handle/11059/5037/65854R741.pdf;jsessionid=7E79A195591B90CD284C980BDD2A9 489? sequence $=1$ 
Rowlands, C. (2006). Just a minute. Works management, 59(11), 19-21.

Shah, R., y Ward, P. (2007). Defining and developing measures of lean production. Journal of operations management, 25(4), 785-805.

Shingo, S. (1985). A revolution in manufacturing: The SMED system. Cambridge y Norwalk, EE. UU.: Productivity Press.

Shingo, S. (1989). A study of the Toyota production system from an industrial engineering viewpoint. Cambridge, EE. UU.: Productivity Press.

Singh, B., y\& Khanduja, D. (2009). SMED: for quick changeovers in foundry SMEs. International journal of productivity and perfomance management, 59(1), 98-116.

Socconini, L. (2008). Lean manufacturing paso a paso: el sistema de gestión empresarial japonés que revolucionó la manufactura y los servicios. [s. d.]: Norma.

Varela, A., Ramírez, J., y Gómez, L. (2015). Lean production system model with Petri nets to support for decision making. Ingeniare, 23(2), 182-195.

Velasco, J. (2013). Organización de la producción. Distribuciones en planta y mejora de los métodos y los tiempos. Madrid, España: Piramide.

Vilana, J. (2010). Fundamentos lean manufacturing. Recuperado de http://api.eoi.es/api_v1_dev. $\mathrm{php} /$ fedora/asset/eoi:75259/componente75258.pdf

Villaseñor, A., y Galindo, E. (2007). Manual de Lean Manufacturing. Guía básica. México, D. F.: Limusa. 
